The Nepali Mathematical Sciences Report, Vol.37 , No.1 \& 2, 2020: 47-54

DOI:https://doi.org/10.3126/nmsr.v37i1-2.34069

\title{
IMPROVED UPPER BOUND TO PRODUCT RATE VARIATION PROBLEM
}

\author{
SHREE RAM KHADKA \\ Central Department of Mathematics, Institute of Science and Technology, Tribhuvan \\ University, Kathmandu, Nepal
}

\begin{abstract}
The sequencing problem which minimizes the deviation between the actual (integral) and the ideal (rational) cumulative production of a variety of models of a common base product is called the product rate variation problem. If the objective is to minimize the maximum deviation, the problem is bottleneck product rate variation problem and the problem with the objective of minimizing all the deviations is the total product rate variation problem.

The problem has been widely studied with several pseudo-polynomial time exact algorithms and heuristics. The lower bound of a feasible solution to the problem has been investigated to be tight. However, the upper bound of a feasible solution had been established in the literature which could further be improved. In this paper, we propose the improved upper bound for BPRVP and TPRVP.

Key Words: Bound, Product rate variation problem, Non-linear integer programming problem
\end{abstract}

AMS (MOS) Subject Classification. 90C47, 91B38.

\section{INTRODUCTION}

The product rate variation problem has been developed when the manufacturing systems switched from mass production to mass customization in order to address customer's demand of a variety of products of a common base product at reasonable prices. The problem addresses sequencing of products in the assembly line, a part of a commonly known Toyota production system [14, 15] in order to eliminate inventories and shortages. The challenge of this just-in-time approach is to prevent shortages while keeping the inventory at a minimum level. Empirical observations have confirmed the positive effects of this approach of mixed-model just-in-time (MMJIT) systems in manufacturing companies [7] and also see [13. The sequence planning is an important issue in mixed-model assembly lines and is closely linked to the complexity of the system [19]. A group decision making approach has been recently proposed in manufacturing systems, [18].

There exist two variants of the sequencing problem of MMJIT systems. One the bottleneck product rate variation problem (BPRVP) with the objective of minimizing the maximum deviation and the other the total product rate variation problem (TPRVP) with the objective of minimizing all the deviations between the actual and the ideal cumulative 
productions [13. Both variants of the problem have strong mathematical base with wide real-world applications, see [3, a survey and therein.

The BPRVP is reduced into a perfect matching problem for a feasible solution and a bisection search algorithm is used for its optimal solution. The solution procedure has been improved with an improved upper bound and the necessary and sufficient condition for the existence of a feasible solution [2]. The PRVP with the objective function other than the absolute deviation has been solved in [4] and [8] by investigating the upper bounds corresponding to the objective functions. The problem with different objective functions yields solution in the same complexity $O(D \log D)$, where $D$ stands for the total demand of all the models, of the problem with an absolute deviation objective [4, 8, 16]. An improved upper bound to the BPRVP has been investigated in [10]. Moreover, the problem with the asymmetric objective function that means different penalty for the inventory and for the shortage has been established [9]. The penalty for tardiness is considered to be higher than the penalty for earliness with the assumption that frequent and extensive shortages are likely to threaten the trust of the customers in the ability of a company to deliver.

The TPRVP is also well studied with exact solution method which is firstly reduced into an assignment problem and is solved in pseudo-polynomial time and also with some heuristics [1, 4, 5, 6, 12, 13, 17]. The complexity of the exact solution method is $D^{3}$, where $D$ is total demand of all copies. The lower and the upper bounds of the TPRVP have also been established, [1]. However, the upper bound can further be improved.

The contribution proposed in this paper can be applied in developing production sequences in the assembly lines in which a variety of models of a common base product is produced. The sequencing approach of production with the implementation of this investigation is exact and based on some assumptions.

Let us consider total demand $D$ of $n, n \geq 2$, different models with $d_{i}$ copies of model $i, i=1,2, \ldots, n$ of a common base product. We assume equal processing time of each model $i, i=1,2, \ldots, n$, in total $D$ equal time units which is called time horizon. A copy of a model is produced in a time unit $k$ means that the copy of the model is produced during the time period from $k-1$ to $k, k=1,2, \ldots, D$. Consider $r_{i}=\frac{d_{i}}{D}$ to be the demand rate and $x_{i k}$ and $k r_{i}, i=1,2, \ldots, n ; k=1,2, \ldots, D$, to be the actual and the ideal cumulative productions, respectively, of model $i$ produced during the time units 1 through $k$. Let us assume equal cost for both inventory $x_{i k}-k r_{i}>0$, and shortage $k r_{i}-x_{i k}>0$. A non linear integer programming formulation for the PRVP, [13, 16] is given below.

Minimize

$$
F_{m}=\max _{i k}\left|x_{i k}-k r_{i}\right|^{m} \quad \text { for BPRVP }
$$

Minimize

$$
\widetilde{F}_{m}=\sum_{k=1}^{D} \sum_{i=1}^{n}\left|x_{i k}-k r_{i}\right|^{m} \quad \text { for TPRVP }
$$

subject to 


$$
\begin{aligned}
\sum_{i=1}^{n} x_{i k} & =k, \quad k=1,2, \ldots, D \\
x_{i(k-1)} & \leq x_{i k}, \quad i=1,2, \ldots, n ; \quad k=2,3, \ldots, D \\
x_{i D} & =d_{i}, x_{i 0}=0, \quad i=1,2, \ldots, n \\
x_{i k} & \geq 0, \text { integer, } \quad i=1,2, \ldots, n ; \quad k=1,2, \ldots, D .
\end{aligned}
$$

In this paper, we propose the improved upper bounds to the BPRVP and the TPRVP both. The remainder of the paper is structured as follows. In Section 2 , we discuss the level curves, lower bound and improved upper bounds for both BPRVP and TPRVP as the main contribution of the paper. The last section concludes the paper.

\section{Bounds}

\section{Level curve}

The actual cumulative production $x_{i k}, i=1,2, \ldots, n ; k=1,2, \ldots, D$, a sequencedependent integer, has $n D$ values with $x_{i k} \in\left\{j \mid j=0,1,2, \ldots, d_{i} ; i=1,2, \ldots, n\right\}$. However, the ideal cumulative production $k r_{i}, i=1,2, \ldots, n ; k=1,2, \ldots, D$, a sequence-independent rational, is such that $k r_{i} \in\left\{\frac{d_{i}}{D}, \frac{2 d_{i}}{D}, \ldots, d_{i}\right\}, i=1, \ldots, n$. Let $j, j=1, \ldots, d_{i}$, be the number of copies of a model $i, i=1,2, \ldots, n$, and $(i, j)$ be the $j^{\text {th }}$ copy of model $i, i=1,2, \ldots, n$. There exist at most $n+D$ different values of $x_{i k}$. Hence, one can replace $x_{i k}$ by $j$ with $j=0,1, \ldots, d_{i} ; i=1,2, \ldots, n$, in the level curve (see Fig. 1) of the objective value of the function. The level curve for copy $(i, j)$ of the objective function of the PRVP is defined as

$$
f_{i j}^{m}=\left|j-k r_{i}\right|^{m}, i=1,2, \ldots, n ; j=0,1, \ldots, d_{i} .
$$

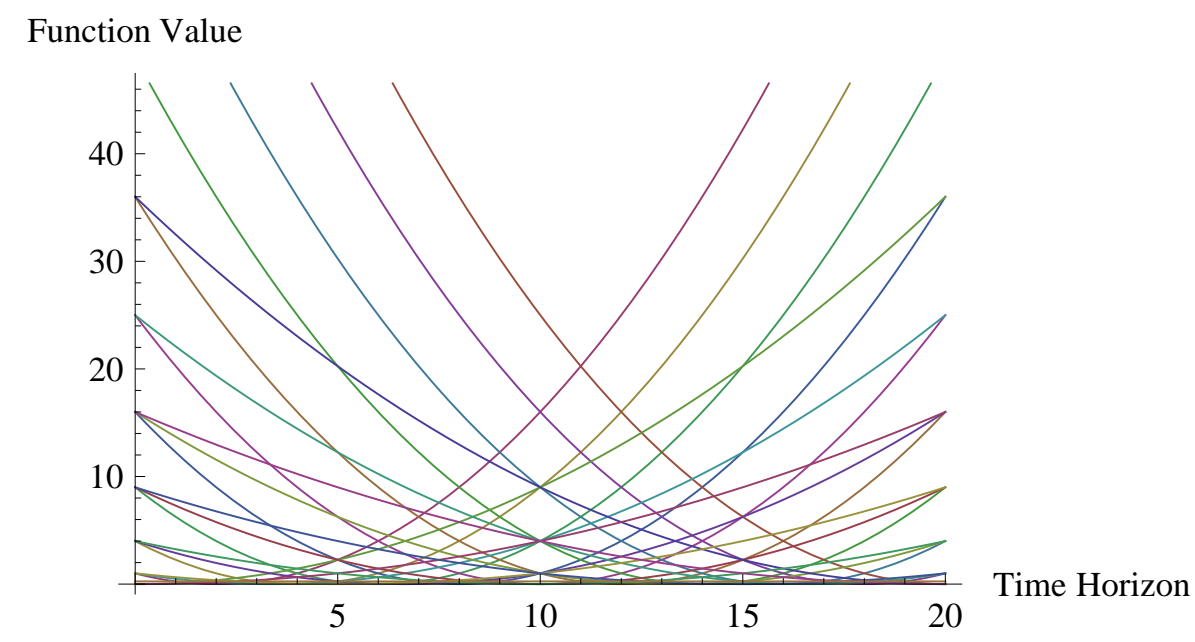

Figure 1. Level curves $f_{i j}^{2}$ for the instance $\left(d_{1}=4, d_{2}=6, d_{3}=10\right)$

The horizontal line with the value $B>0$ intersecting the level curve for each copy $(i, j), i=1,2, \ldots, n ; j=1,2, \ldots, d_{i}$, is called a bound for the problem. 


\section{Lower bound}

The importance of a lower bound $L B_{m}$ on the optimal value of function $F_{m}$ for the problem results from the fact that a feasible sequence $s$ of an instance $\left(d_{1}, d_{2}, \ldots, d_{n}\right)$ obtained with an objective function value equal to the lower bound is optimal. However, not all instances are even feasible at this value. The tight lower bound for BPRVP has been investigated to be $L B_{m}=\left(1-r_{\max }\right)^{m}$, see [4, 8]. It is note-worthy that a lower bound on the absolute deviation objective function for the BPRVP has been established by Steiner and Yeomans [16]. Likewise the lower bound for TPRVP has been investigated to be $L B_{m}=n D\left(1-r_{\max }\right)^{m}$, see [11].

\section{Upper bound}

An upper bound $U B_{m}, m$ being a positive integer, must satisfy the following two inequalities which are the necessary and sufficient condition for the existence of a feasible sequence for the BPRVP with the objective function $F_{m}$,

$$
\sum_{i=1}^{n}\left(\left\lfloor k_{2} r_{i}+\sqrt[m]{U B_{m}}\right\rfloor-\left\lceil\left(k_{1}-1\right) r_{i}-\sqrt[m]{U B_{m}}\right\rceil\right) \geq k_{2}-k_{1}+1
$$

and

$$
\sum_{i=1}^{n}\left(\left\lceil k_{2} r_{i}-\sqrt[m]{U B_{m}}\right\rfloor-\left\lfloor\left(k_{1}-1\right) r_{i}+\sqrt[m]{U B_{m}}\right\rfloor\right) \leq k_{2}-k_{1}+1
$$

with $k_{1}, k_{2} \in\{1, \ldots, D\}, k_{1} \leq k_{2}$, see Khadka [8]. If $m=1$, the two inequalities become the necessary and sufficient condition for the problem with the objective function $F_{1}$, see Brauner and Crama [2].

Initially the upper bound $U B_{1}$ to the problem BPRVP has been investigated to be $U B_{1}=1$, [16] and later it has been improved to be $U B_{1}=1-\frac{1}{D}$ for the problem with the objective function $F_{1}$, 2] and to be $U B_{m}=\left(1-\frac{1}{D}\right)^{m}$ for the problem with the objective function $F_{m}$, [8]. The upper bound $U B_{m}=\left(1-\frac{1}{D}\right)^{m}$ can be improved to be $U B_{m}=$ $\left(1-r_{\min }\right)^{m}$, where $r_{\min }=\frac{d_{\min }}{D}$, in the case of an instance $\left(d_{1}, d_{2}, \ldots, d_{n}\right)$ with $d_{\min } \geq 1$, $\min _{i}\left(d_{i}\right)=d_{\text {min }}, i=1,2, \ldots, n$.

\section{Theorem 2.1. Let}

$$
U B_{m}=\left(1-r_{\text {min }}\right)^{m}, \text { where } r_{\text {min }}=\frac{d_{\text {min }}}{D}, \min _{i}\left(d_{i}\right)=d_{\text {min }}, i=1,2, \ldots, n .
$$

Then $U B_{m}$ is an upper bound on the largest value of the objective function $F_{m}$ of a feasible solution for BPRVP.

Proof. Let us consider a general instance $\left(d_{1}, d_{2}, \ldots, d_{n}\right)$ with $d_{\min } \geq 1$, where $\min _{i}\left(d_{i}\right)=$ $d_{\text {min }}, i=1,2, \ldots, n$. If $U B_{m}$ is an upper bound on the largest value of the objective function $F_{m}$ of a feasible solution for the BPRVP, then this bound $U B_{m}$ satisfies the inequality

$$
\left|x_{i k}-k r_{i}\right|^{m} \leq U B_{m}
$$

for all $i=1,2, \ldots, n ; k=1,2, \ldots, D$, for any feasible solution $s \in \mathcal{X}$. 
We claim that the improved upper bound on the largest value of the objective function $F_{m}$ is

$$
U B_{m}=\left(1-r_{\min }\right)^{m}
$$

where

$$
\min _{i}\left(r_{i}\right)=r_{\min }=\frac{d_{\min }}{D}, i=1,2, \ldots, n
$$

We need to show the proposed upper bound (2.4) satisfying the two inequalities (2.1) and 2.2 .

We can write

$$
\begin{aligned}
\left\lfloor k_{j} r_{i}+\sqrt[m]{U B_{m}}\right\rfloor & =\left\lfloor k_{j} r_{i}+1-r_{\text {min }}\right\rfloor . \\
& =\left\lfloor k_{j} r_{i}+1-\frac{d_{\text {min }}}{D}\right\rfloor, i=1,2, \ldots, n ; j=1,2 .
\end{aligned}
$$

If $k_{j} r_{i}$ is an integer,

$$
\left\lfloor k_{j} r_{i}+1-\frac{d_{\text {min }}}{D}\right\rfloor=k_{j} r_{i}, i=1,2, \ldots, n ; j=1,2,
$$

and if $k_{j} r_{i}$ is not an integer,

$$
k_{j} r_{i}=\left\lfloor k_{j} r_{i}\right\rfloor+\left(k_{j} r_{i}\right)_{\text {frac }}, i=1,2, \ldots, n ; j=1,2,
$$

where $\left(k_{j} r_{i}\right)_{\text {frac }}$ is the fractional part of $k_{j} r_{i}$.

Since $\frac{d_{\min }}{D} \leq\left(k_{j} r_{i}\right)_{\text {frac }} \leq 1-\frac{d_{\min }}{D}$,

$$
\begin{aligned}
\left\lfloor k_{j} r_{i}+1-r_{\text {min }}\right\rfloor & =\left\lfloor\left\lfloor k_{j} r_{i}\right\rfloor+\left(k_{j} r_{i}\right)_{\text {frac }}+1-\frac{d_{\text {min }}}{D}\right\rfloor \\
& \geq\left\lfloor k_{j} r_{i}\right\rfloor+1 \\
& >k_{j} r_{i}, i=1,2, \ldots, n ; j=1,2 .
\end{aligned}
$$

Therefore,

$$
\left\lfloor k_{j} r_{i}+\sqrt[m]{U B_{m}}\right\rfloor \geq k_{j} r_{i}, i=1,2, \ldots, n ; j=1,2 .
$$

Likewise,

$$
\begin{aligned}
\left\lceil k_{j} r_{i}-\sqrt[m]{U B_{m}}\right\rceil & =\left\lceil k_{j} r_{i}-1+r_{\min }\right\rceil \\
& =\left\lceil k_{j} r_{i}-1+\frac{d_{\min }}{D}\right\rceil, i=1,2, \ldots, n ; j=1,2
\end{aligned}
$$

If $k_{j} r_{i}$ is an integer,

$$
\left\lceil k_{j} r_{i}-1+\frac{d_{\text {min }}}{D}\right\rceil=k_{j} r_{i}, i=1,2, \ldots, n ; j=1,2,
$$

and if $k_{j} r_{i}$ is not an integer,

$$
\begin{aligned}
\left\lceil k_{j} r_{i}-1+\frac{d_{\text {min }}}{D}\right\rceil & =\left\lceil\left\lfloor k_{j} r_{i}\right\rfloor+\left(k_{j} r_{i}\right)_{\text {frac }}-1+\frac{d_{\text {min }}}{D}\right\rceil \\
& \leq\left\lceil\left\lfloor k_{j} r_{i}\right\rfloor\right\rceil \\
& <k_{j} r_{i}, i=1,2, \ldots, n ; j=1,2 .
\end{aligned}
$$


Therefore,

$$
\left\lceil k_{j} r_{i}-\sqrt[m]{U B_{m}}\right\rceil \leq k_{j} r_{i}, i=1,2, \ldots, n ; j=1,2 .
$$

Hence, using the inequalities 2.5 and 2.6,

$$
\begin{aligned}
\sum_{i=1}^{n}\left(\left\lfloor k_{2} r_{i}+\sqrt[m]{U B_{m}}\right\rfloor-\left\lceil\left(k_{1}-1\right) r_{i}-\sqrt[m]{U B_{m}}\right\rceil\right) & \geq \sum_{i=1}^{n} k_{2} r_{i}-\sum_{i=1}^{n}\left(k_{1}-1\right) r_{i} \\
& \geq k_{2}-k_{1}+1
\end{aligned}
$$

And,

$$
\begin{aligned}
\sum_{i=1}^{n}\left(\left\lceil k_{2} r_{i}-\sqrt[m]{U B_{m}}\right\rceil-\left\lfloor\left(k_{1}-1\right) r_{i}+\sqrt[m]{U B_{m}}\right\rfloor\right) & \leq \sum_{i=1}^{n} k_{2} r_{i}-\sum_{i=1}^{n}\left(k_{1}-1\right) r_{i} \\
& \leq k_{2}-k_{1}+1
\end{aligned}
$$

This shows that

$$
U B_{m}=\left(1-r_{\min }\right)^{m}
$$

satisfies the two inequalities 2.1 and 2.2 proving to be the upper bound of a feasible solution for the BPRVP with the objective function

$$
\max _{i, k}\left|x_{i k}-k r_{i}\right|^{m}, i=1,2, \ldots, n ; k=1,2, \ldots, D .
$$

In particular, for any instance $\left(d_{1}, d_{2}, \ldots, d_{n}\right)$ with $d_{\min }=1$, the upper bound,

$$
U B_{m}=\left(1-r_{\min }\right)^{m},
$$

is exactly the same as the upper bound established in 22 for the problem with the objective function $F_{1}$ and, in [8] for the objective function $F_{m}, m$ being a positive integer.

The improved upper bound for TPRVP can be established to be

$$
U B_{m}=n D\left(1-r_{m i n}\right)^{m}
$$

, which has been proven below.

Theorem 2.2. Let

$$
\widetilde{U B}_{m}=n D\left(1-r_{m i n}\right)^{m}, r_{m i n}=\frac{d_{m i n}}{D}, \min \left(d_{i}\right)=d_{m i n}, i=1,2, \ldots, n .
$$

Then $\widetilde{U B}_{m}$ is an upper bound on the largest value of the objective function $\widetilde{F}_{m}$ of a feasible solution for TPRVP.

Proof. If $\widetilde{U B}_{m}$ is an upper bound on the largest value of the objective function $\widetilde{F}_{m}$ of a feasible solution for TPRVP, then this bound $\widetilde{U B}_{m}$ satisfies the inequality

$$
\sum_{k=1}^{D} \sum_{i=1}^{n}\left|x_{i k}-k r_{i}\right|^{m} \leq \widetilde{U B}_{m}
$$

for any feasible solution $\mathrm{x} \in \mathcal{X}$.

It has been established that the improved upper bound is $\left(1-r_{\min }\right)^{m}$ for BPRVP with the objective function $F_{m}$. 
Now, we obtain

$$
\begin{aligned}
\sum_{k=1}^{D} \sum_{i=1}^{n}\left|x_{i k}-k r_{i}\right|^{m} & =\sum_{i=1}^{n}\left|x_{i 1}-1 r_{i}\right|^{m}+\cdots+\sum_{i=1}^{n}\left|x_{i D}-D r_{i}\right|^{m} \\
& =\left|x_{11}-1 r_{1}\right|^{m}+\cdots+\left|x_{n 1}-1 r_{n}\right|^{m}+\ldots \\
& +\left|x_{1 D}-D r_{1}\right|^{m}+\cdots+\left|x_{n D}-D r_{n}\right|^{m} \\
& \leq n D \cdot \max _{i, k}\left|x_{i k}-k r_{i}\right|^{m} \\
& \leq n D\left(1-r_{\min }\right)^{m} .
\end{aligned}
$$

Hence, an upper bound $\widetilde{U B}_{m}$ on the largest value of the objective function $\widetilde{F}_{m}$ of a feasible solution for TPRVP is given by

$$
\widetilde{U B}_{m}=n D\left(1-r_{m i n}\right)^{m}
$$

\section{Concluding Remarks}

We considered the product rate variation problem with the unimodular symmetric objective function having zero at zero. It has two variants one the problem which is called the bottleneck product rate variation problem with the objective $F_{m}, m$ being a positive integer, of minimizing its maximum deviation between the actual and the ideal cumulative production of a variety of models of a common base product which has been solved with an exact solution having its complexity $D \log D, D$ being the total demand. The other variant is known as total product rate variation problem with the objective function $\widetilde{F}_{m}$ of minimizing all the deviations instead of its maximum deviation. This problem has been solved with many heuristics and an exact solution with complexity $D^{3},[12]$.

A lower bound $1-r_{\max }$ of a feasible solution of BPRVP with the objective function $F_{1}$, [16], and $\left(1-r_{\max }\right)^{m}$ for the objective function $F_{m}, m$ being a positive integer, [8], and an upper bound $1-\frac{1}{D}$ for the objective function $F_{1},[2]$, and $\left(1-\frac{1}{D}\right)^{m},[8]$, for the objective function $F_{m}$ of a feasible solution for the BPRVP have been established.

Likewise, a lower bound and an upper bound of a feasible solution of TPRVP $n D(1-$ $\left.r_{\max }\right)^{m}$ and $n D\left(1-\frac{1}{D}\right)^{m}, m$ being a positive integer investigated in [11].

In this paper, we have proposed improved upper bounds to be $\left(1-r_{\min }\right)^{m}$ and $n D(1-$ $\left.r_{\text {min }}\right)^{m}$ for BPRVP and TPRVP, respectively. Implementation of the improved upper bounds while applying the exisitng algorithms to find optimal solution would be interesting in the future work.

\section{REFERENCES}

[1] N. Boysen, M. Fliedner and Scholl A, Sequencing mixed-model assembly lines: Survey, classification and model critique, European Journal of Operations Research Vol. 192 (2), pp 349-373, 2009.

[2] N. Brauner and Y. Crama, The maximum deviation just-in-time scheduling problem, Discrete Applied Mathematics, Vol. 134 (1), pp 25-50, 2004. 
[3] T. N. Dhamala and S. R. Khadka, A review on sequencing approaches for mixed-model just-in-time production system, Iranian Journal of Optimization, Vol. 1 (3), pp 266-290, 2009.

[4] T. N. Dhamala, S. R. Khadka and M. H. Lee, A note on bottleneck product rate variation problem with square-deviation objective, International Journal of Operations Research, Vol. 7 (1), pp 1-10, 2010.

[5] T. N. Dhamala and W. Kubiak, A brief survey of just-in-time sequencing for mixed-model systems, International Journal of Operations Research Vol. 2, (2), pp 38-47, 2005.

[6] E. Duplaga and D. Bragg, Mixed-model assembly line sequencing heuristics for smoothing component parts usage: a comparative analysis, International Journal of Production Research, Vol. 36 (8), pp 2209-2224, 1998.

[7] M. Huson and D. Nanda, The impact of just-in-time manufacturing on firm performance in the US, Journal of Operations Management, Vol. 12 (3,4), pp 297 - 310,1995.

[8] S. R. Khadka, Mixed-model just-in-time sequencing problem, Lambert Academic Publishing (LAP), Germany, 2012.

[9] S. R. Khadka, Optimality of the Bottleneck Product Rate Variation Problem with Asymmetric Objective Function, International Journal of Operational Research/Nepal, Vol. 7 (1), pp 43-54, 2018.

[10] S. R. Khadka and T. Becker, On Upper Bound for the Bottleneck Product Rate Variation Problem, Springer International Publishing Switzerland, M. Freitag et al. (eds.), Dynamics in Logistics, Lecture Notes in Logistics, pp 391-399, 2017.

[11] S.R. Khadka and F. Werner, Upper and lower bounds for the total product rate variation problem, Neural, Parallel and Scientific Computations, Vol. 22 (3), pp 389- 398, 2014.

[12] W. Kubiak, Minimizing variation of production rates in just-in-time systems: A survey, European Journal of Operational Research Vol. 66, pp 259-271, 1993.

[13] J. Miltenburg, Level schedules for mixed-model assembly lines in just-in-time production systems, Management Science, Vol. 35 (2), pp 192-207, 1989.

[14] Y. Monden, Toyota production system: an integrated approach to just-in-time, CRC Press, 2011.

[15] T. Ōhno, Toyota production system: beyond large-scale production, Productivity Press, 1988.

[16] G. Steiner and S. Yeomans, Level schedules for mixed-model, just-in-time processes, Management Science, Vol. 39 (6), pp 728-735, 1993.

[17] M. Yavuz and E. Akacali, Production smoothing in just-in-time manufacturing systems: a review of the models and solution approaches, International Journal of Production Research Vol. 45, pp 3579-3597, 2007.

[18] C. Yu, A. Matta and Q. Semeraroi, Group decision making in manufacturing systems: An approach using spatial preference information and indifference zone, Journal of Manufacturing Systems Vol. 55, pp $109-125,2020$.

[19] X. Zhu, S. J. Hu, Y. Koren and N. Huang, A complexity model for sequence planning in mixed-model assembly lines, Journal of manufacturing systems, Vol. 31, pp 121-130, 2012. 